\title{
Algorithm for the management of ectropion through medial and lateral canthopexy
}

\author{
Siook Baek, Jae-Ho Chung, Eul-Sik Yoon, Byung-Il Lee, Seung-Ha Park \\ Department of Plastic and Reconstructive Surgery, Korea University College of Medicine, Seoul, Korea
}

Background Patients with ectropion experience devastating symptoms. Therefore, the prevention and management of this condition are of utmost importance. To treat ectropion, it is important to perform medial and lateral canthopexy in an effective way. In this study, we propose a comprehensive algorithm for the prevention and management of ectropion based on a new classification of ectropion according to its signs and causes.

Methods Canthopexy was performed in 68 cases according to the proposed algorithm, which starts with a categorization of the types of ectropion and ends with the recommended operative technique. To assess the results, we reviewed clinical preoperative and postoperative photographs. To evaluate improvements in patients' symptoms, we conducted a survey with responses scored on a Likert scale.

Results None of the patients had scleral show postoperatively. The average patient satisfaction score was satisfied or higher for all symptoms, and the most improved symptom was aesthetic appearance. No major complications were reported.

Conclusions For the comprehensive management of ectropion, it is crucial to consider both treatment and prevention. Through the simple surgical algorithm proposed in this study, both medically acceptable results and high levels of patient satisfaction were achieved without significant postoperative complications. We recommend using this algorithm for the comprehensive management of ectropion.

Keywords Ectropion / Eyelids / Blepharoplasty
Correspondence: Seung-Ha Park Department of Plastic and Reconstructive Surgery, Korea University College of Medicine, 73 Inchon-ro, Seongbuk-gu, Seoul 02841 , Korea

Tel: +82-2-920-5368

Fax: +82-2-922-7437

E-mail: shp98@korea.ac.kr

\section{INTRODUCTION}

Ectropion is a common problem seen by plastic surgeons in the clinic, and its adequate prevention and management are crucial. Ectropion is a medical condition in which the eyelid rolls away from the globe [1]. Patients with ectropion typically complain of irritable dry eyes and symptoms such as epiphora, keratopathy, lagophthalmos, and pain [2].

Ectropion is traditionally classified into three broad categories according to its pathophysiology: paralytic, cicatricial, and involutional [3]. Paralytic ectropion is caused by a flaccid orbicularis oculi, resulting in loosening of the muscular sling that supports the lid against gravity. Gravity continuously pulls the lower lid downward, and it eventually rotates away. In cicatricial ectropion, a scar contracture or deformity formed by trauma on the anterior lamella acts as the main retracting force on the lower lid. Involutional (atrophic) ectropion, also known as senile ectropion, occurs when the aging process results in atrophy of muscular 
and tendon structures, and the lid becomes abnormally lax and falls away from the orbit.

However, we suggest that in addition to clinically evident ectropion, potential ectropion should also be managed in order to prevent it from manifesting. Some patients may have sufficient lid laxity to develop ectropion in the future, even if they do not present with overt features of ectropion. Furthermore, patients preparing to undergo procedures that can cause secondary ectropion should receive prophylactic treatment. Therefore, we developed a more practical and comprehensive categorization of ectropion.

Various surgical procedures are used to treat lower lid ectropion $[2,4-6]$. By evaluating the types of ectropion preoperatively, the most appropriate technique for each patient should be selected and performed; however, there is no established surgical algorithm. Several previous studies have proposed approaches for lower lid ectropion, but they were generally based on the three types of ectropion in the conventional classification, and included procedures that are too extensive and aggressive [7-9].

For the comprehensive management of ectropion, we propose a simple algorithm based on a new classification of types of ectropion according to its signs and causes. This will help surgeons to classify and manage each type of ectropion with appropriate procedures.

\section{METHODS}

\section{Patients}

Institutional review board/ethics committee approval was obtained from the Institutional Review Board of Korea University Anam Hospital (K2018-1711-003). Medical records and preoperative and postoperative clinical photographs of patients who underwent canthopexy procedures were reviewed retrospectively. A total of 68 canthopexy procedures were performed between January 2014 and March 2018 at our center.

We categorized ectropion as "potential ectropion" OR "latent ectropion" and "current ectropion" OR "present ectropion" in our algorithmic approach, using the following criteria: (1) preoperative evaluations: snap-back test, distraction test; (2) photographic analysis: scleral show, lid eversion, lid malposition; or (3) chief complaint and symptoms of patients.

As suggested by the names of these categories, patients who did not have evident ectropion features, but in whom ectropion had the potential to develop, were defined as having potential or latent ectropion. In patients with this type of ectropion, the degree of laxity determined the type of canthopexy procedure. Lid laxity was evaluated by a preoperative physical examination. Scleral show with lid eversion and/or malposition was defined
Table 1. Patient demographics

\begin{tabular}{|llcccc|}
\hline Type & \multicolumn{1}{c}{ Cause } & Right & Left & Bilateral & $\begin{array}{r}\text { Total } \\
\text { cases }\end{array}$ \\
\hline $\begin{array}{l}\text { Present or current } \\
\text { ectropion } \\
\quad \text { Paralytic }\end{array}$ & 13 & 17 & 1 & 32 \\
& Postoperative & 5 & 7 & 0 & 12 \\
Cicatricial & Bell palsy & 2 & 2 & 0 & 4 \\
& Trauma & 5 & 8 & 0 & 13 \\
& Postoperative & 0 & 0 & 1 & 2 \\
Potential or latent & Burn & 1 & 0 & 0 & 1 \\
ectropion & & 0 & 0 & 18 & 36 \\
Total cases & & 13 & 17 & 38 & 68 \\
\hline
\end{tabular}

as current or present ectropion. In this category of patients, lid malposition was the decisive criterion for management.

A total of 36 cases of potential or latent ectropion and 32 cases of present or current ectropion were enrolled (Table 1), in 29 female and 20 male patients. The mean age of the patients was 51.6 \pm 13.1 years (range, $20-76$ years), and the mean follow-up period was 18.71 weeks (range, 6-72 weeks).

\section{Preoperative evaluations}

First, we reviewed each patient's medical history and carefully examined the periorbital appearance to evaluate the presence of eyelid retraction, scleral show, and keratopathy. Subsequently, distraction and snap-back tests were performed to assess lid tone. Patients who had more than $6 \mathrm{~mm}$ of anterior distraction were considered to have severe lid laxity [9].

If the patient had associated facial palsy symptoms, other diagnostic examinations such as electromyography and physical examinations were performed as facial expression assessments to confirm the involvement of the central or peripheral nervous system and to evaluate the presence of complete or incomplete palsy, in order to determine whether additional operations were necessary.

\section{Operative techniques}

All patients underwent canthopexy procedures according to their preoperative evaluation results (Fig. 1). The senior author (S.H.P.) performed lateral canthopexies in a total of 60 cases. Bone drilling fixation was performed in four cases, tarsal strip suspension in 20 cases, and orbicularis oculi muscle suspension in the remaining 36 cases. Eight medial canthopexies were performed and in all cases, Mitek bone anchoring was performed. The preoperative design was done before the administration of anesthesia in an upright seated position; for vasoconstriction, lidocaine mixed with epinephrine was injected into the predicted incision and dissection areas. 


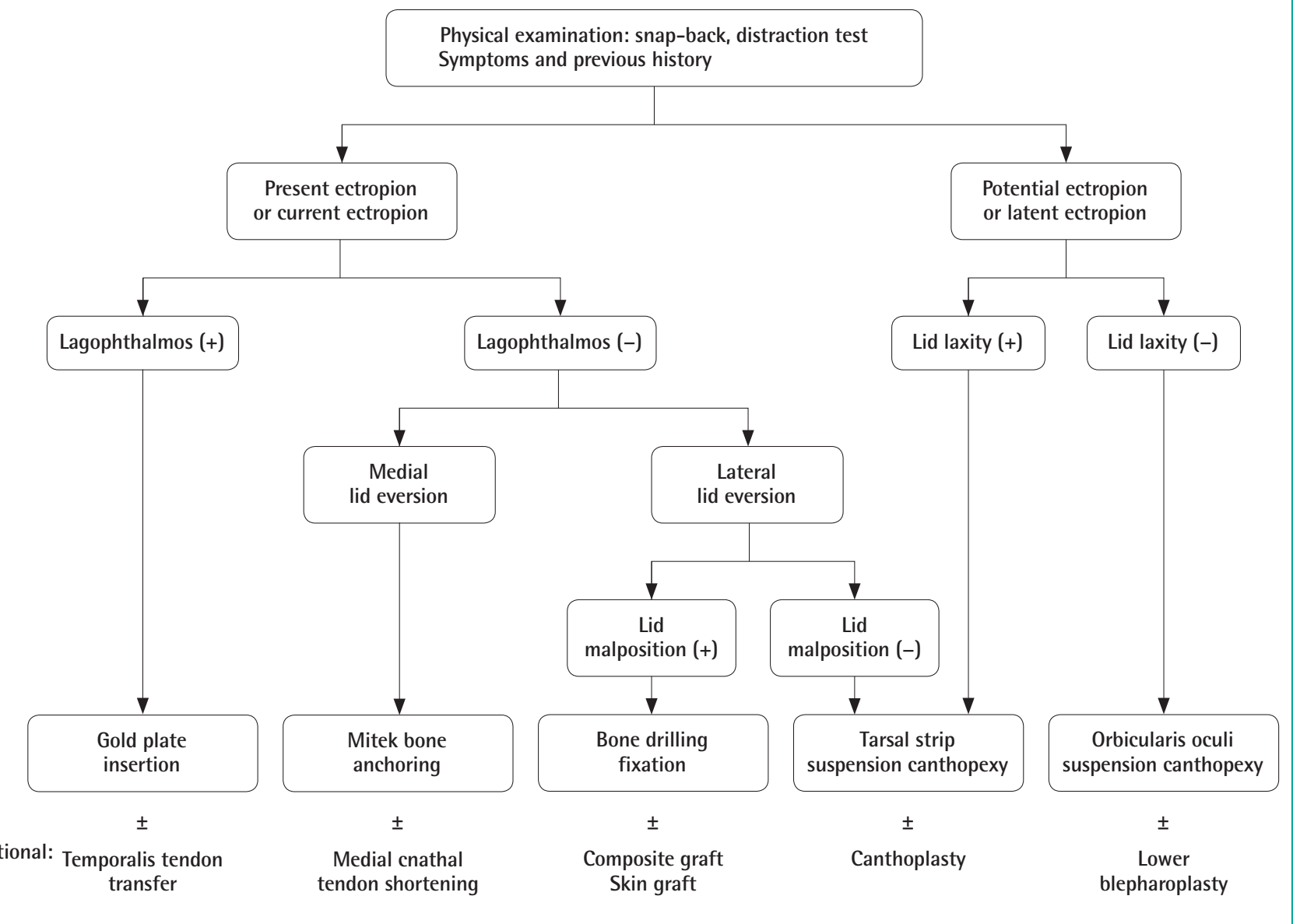

Potential or latent ectropion

Potential or latent ectropion without lid laxity: orbicularis oculi muscle suspension

Generally, the patients included in this category underwent lower blepharoplasty and had thin lower lid skin. Canthopexy was performed as a preventive procedure for potential ectropion.

We performed routine lower eyelid blepharoplasty procedures and additional canthopexy procedures with orbicularis oculi muscle suspension. Doing so had several important advantages in addition to the main preventive effect of canthopexy. A significant rejuvenating effect was obtained by augmenting the pretarsal area and by tightening bulging of the orbital septum. After the subciliary incision was made, we split the orbicularis muscle $7 \mathrm{~mm}$ inferior to the subciliary margin in order to preserve pretarsal muscle function. Modulation of the orbital septum and fat was performed if needed. The anchor site of the orbicularis oculi muscle was identified and bluntly dissected to the lateral orbital rim. The lateral edge of the preseptal orbicularis muscle was suspended to the periosteum of the anchoring point with Vicryl 5-0 or 6-0 (Ethicon, Inc., Somerville, NJ, USA) with appropri- ate tension by a horizontal mattress suture.

In patients with excessive skin, the remaining skin was resected after redraping. The incision sites were closed with subcutaneous Vicryl 6-0 and Prolene 6-0 (Ethicon, Inc.) skin sutures.

Potential or latent ectropion with lid laxity: lateral tarsal strip suspension

In patients who had no evident ectropion but did show lid laxity, the tarsal strip was used as the target of suspension through wedge resection. We primarily performed 3 to 5-mm full-thickness wedge resection at the lateral canthal angle. This junction corresponded to the most lateral aspect of the lower lid margin. By isolating the lateral tarsal stump end to free it from the surrounding skin and conjunctiva using tenotomy scissors, the tarsal strip was prepared for suspension (Fig. 2A).

The anchoring sites in this group differed from those previously described. The tarsal strip was fixated $5 \mathrm{~mm}$ superior to the most lateral side of the lateral orbital rim using an Ethibond 5-0 suture (Ethicon, Inc.) (Fig. 2B). The conjunctiva and subcutaneous tissues were closed with Vicryl 6-0 sutures and the 


\section{Fig. 2. Lateral tarsal strip suspension canthopexy}

(A) A 3 to 5-mm full-thickness wedge resection at the junction of the tarsus and lateral canthal tendon. Isolation of the medial tarsal stump end to free it from surrounding skin and conjunctiva. (B) The targeted anchoring point was $5 \mathrm{~mm}$ superior to the most lateral side of the lateral orbital rim. The tarsal strip was fixated to the anchoring point.

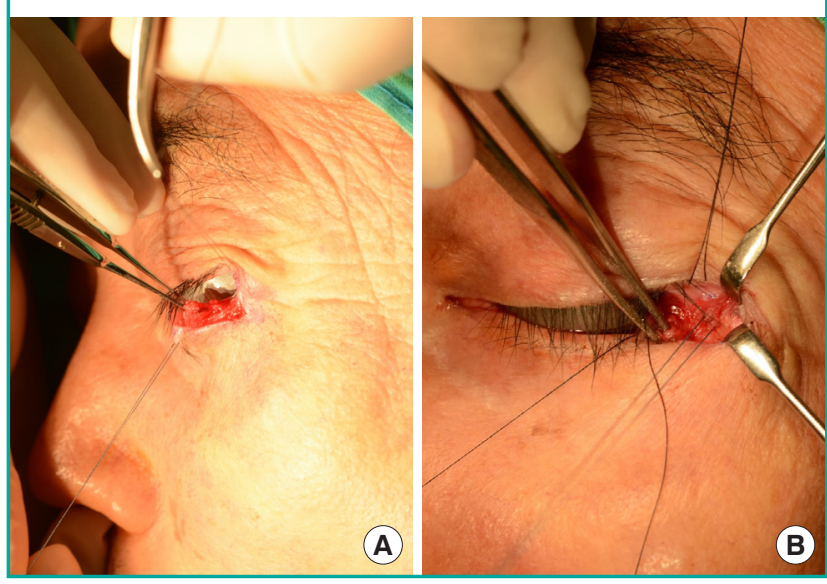

skin was primarily closed with Prolene 6-0 sutures.

\section{Current or present ectropion}

In this category of patients, the lateral tarsal strip was the target for the suspension procedure.

Current or present ectropion without lateral lid malposition: lateral tarsal strip suspension

If the patient only had lateral lid eversion, lateral tarsal strip canthopexy was performed, which is the same procedure performed in cases of potential ectropion with lid laxity, as discussed above. If they had lid laxity, additional canthoplasty was performed.

Current or present ectropion with lateral lid malposition: lateral orbital bone drilling

In patients who had lateral lid eversion with lash malposition, resulting in lateral canthal tenting above the globe, the procedure was the same as in those with lateral tarsal strip canthopexy, except for a difference in the target for anchoring and the material used for suspension. First, the bone was used as the anchoring spot. Second, steel 1-0 wire or Ethibond 4-0 was used as the material for suspension. Bone drilling was necessary on the medial side of the lateral orbital rim $5 \mathrm{~mm}$ superior to Whitnall tubercle for the passage of the wire (Fig. 3).

Current or present ectropion with medial lid malposition: medial canthopexy using Mitek bone anchoring

In ectropion patients with medial lacrimal punctum eversion and
Fig. 3. Lateral tarsal strip fixation through bone drilling

(A) After drilling the lateral orbital wall, the steel wire passes through the hole from the inner to outer aspects of the lateral rim. (B) The lateral tarsal end was anchored to a more inner position, similar to the original position of Whitnall tubercle.
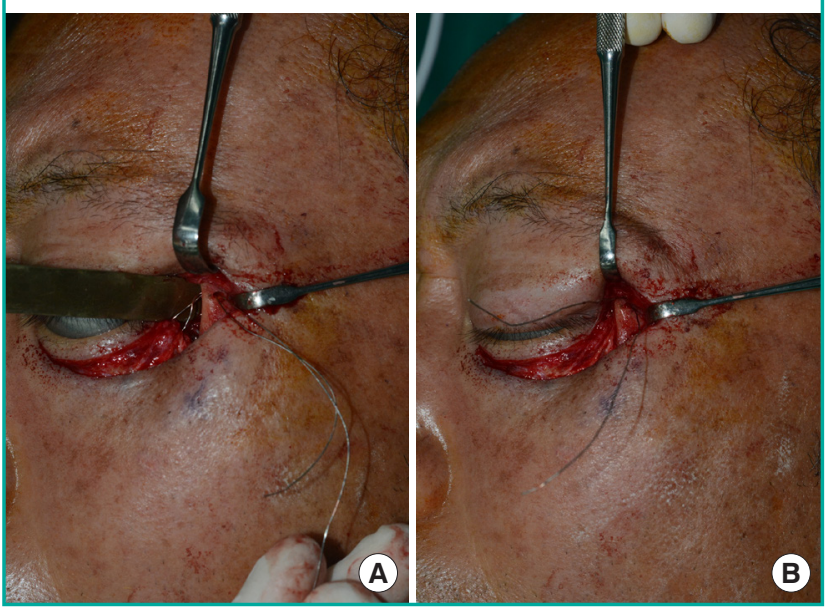

medial canthal widening, medial canthopexy was performed. The medial orbital rim was accessed through a transcutaneous incision below the subciliary and lacrimal punctum and extended to the medial side. A tenotomy scissor was used to bluntly dissect this area to prevent lacrimal apparatus injuries (Fig. 4A). Through the window, the medial canthal tendon was exposed. The anchoring spot of medial canthopexy was placed just superior to the crossing point of the anterior and posterior lacrimal crest (Fig. 4B). The material used for the suspension was Mitek (Mitek QuickAnchor; Mitek Surgical Products, Inc., Westwood, MA, USA) (Fig. 4C and D). If there was severe laxity of the medial canthal tendon, an additional shortening procedure was done.

Current or present ectropion with lamellar contracture: skin graft or composite graft

If severe contracture was found on the anterior or posterior lamella, an additional procedure was performed. In cases of anterior lamellar contracture, a skin graft was used. For posterior lamellar contracture, a composite graft such as a concha cartilage or palatal mucosal graft was used.

\section{Lagophthalmos on the upper eyelid: gold plate insertion}

If a patient had ectropion and separate upper eyelid lagophthalmos, a gold plate was inserted on the levator aponeurosis and tarsal plate of the upper eyelid. Generally, most patients with upper eyelid lagophthalmos had issues with facial palsy. Based on the preoperative examination for facial palsy, patients with irreversible facial palsy but intact temporalis function could undergo temporalis tendon transfer surgery for facial reanimation. 


\section{Fig. 4. Medial canthopexy using Mitek bone anchor system}

(A) The medial orbital rim was accessed through a small transcutaneous incision. (B) The medial canthal tendon could be identified and the anchoring point of suspension was set just above to the medial canthal tendon insertion site. For the exposure of periosteum on the anchoring point, it was bluntly dissected, with care taken to prevent lacrimal apparatus injuries. (C) The Mitek system was applied. (D) The lateral tarsal string was suspended and fixed with adequate tension.
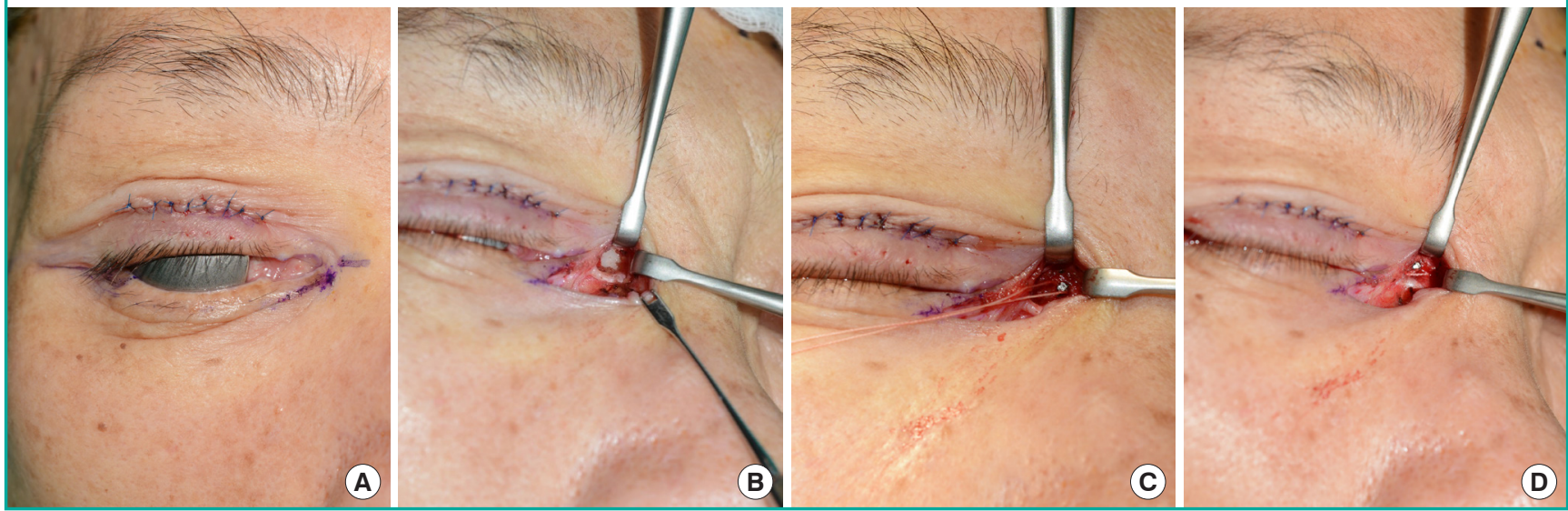

In patients with eyebrow ptosis, brow lift procedures were also performed.

\section{Assessment of results}

We reviewed preoperative and postoperative clinical photographs to evaluate scleral show, lid eversion, and malposition. We also analyzed satisfaction scores from patients with current or present ectropion using a 1 to 5 scale with responses of 1 (poor), 2 (borderline), 3 (satisfied), 4 (good), and 5 (excellent) in order to evaluate the improvement of subjective symptoms. The symptoms evaluated in the survey included epiphora, dryness, pain or irritation, and aesthetic appearance. Postoperative complications such as infection, secondary ectropion (recurrence), or entropion were also reviewed.

\section{RESULTS}

The mean patient satisfaction score after surgery regarding symptoms was $3.47 \pm 0.98$ on a scale of 1 to 5 , and the most improved symptom was aesthetic appearance, for which the average score was $4.22 \pm 0.79$. The least improved symptom was epiphora, with an average score of $2.89 \pm 0.94$.

Postoperatively, none of the patients had scleral show, lid eversion, or malposition. The following clinical photographs represent different types of ectropion corrected using the appropriate surgical procedures.

\section{Case 1: Current cicatricial ectropion without lateral lid malposition}

A 76-year-old male patient presented with unilateral secondary ectropion caused by previously performed lower blepharoplasty. The patient showed bilateral lateral lid eversion without malposition. On both sides, we performed lateral canthopexy using a tarsal strip suspension suture. No additional grafting procedure was needed since there was minimal lamellar contracture. After 3 months, on both sides, scleral show had resolved, and the externally exposed lashes had disappeared. Lower lid laxity decreased bilaterally after wedge resection (Fig. 5).

\section{Case 2: Current cicatricial ectropion with lateral lid malposition}

A 61-year-old male patient had a history of infectious disease resulting in a destructive deformity on a unilateral bony orbit and surrounding soft tissues. This deformity caused severe ectropion with lateral lid malposition. He primarily underwent reconstructive surgery for the orbital bone defect using high-density porous polyethylene (Medpor; Porex Technologies Corporation, Fairburn, GA, USA). A combined lateral canthopexy procedure using 1-0 steel-wire suspension to the lateral orbital rim with drilling and a full-thickness skin graft to the anterior lamella was performed. This combined procedure was necessary to resolve the patient's severe anterior lamellar contracture. After 2 years, ectropion recurred, and tarsal strip suspension was implemented in a revisional procedure. After 1 year of follow-up after the last operation, the result remained stable and corrected (Fig. 6).

\section{Case 3: Current paralytic ectropion with medial and lateral lid malposition}

A 69-year-old female patient had severe medial and lateral lid malposition caused by congenital unilateral complete facial pal- 


\section{Fig. 5. Cicatricial ectropion without lateral lid malposition}

(A) A 76-year-old male patient with unilateral secondary ectropion caused by a previously performed lower blepharoplasty. The patient showed bilateral lateral lid eversion without malposition. On both sides, we performed lateral canthopexy using a tarsal strip suspension suture with the Mitek bone anchor system. (B) After 3 months, scleral show had resolved on both sides. There was secure contact of the eyelid margin with the eyeball. The lower lid laxity decreased bilaterally after wedge resection.
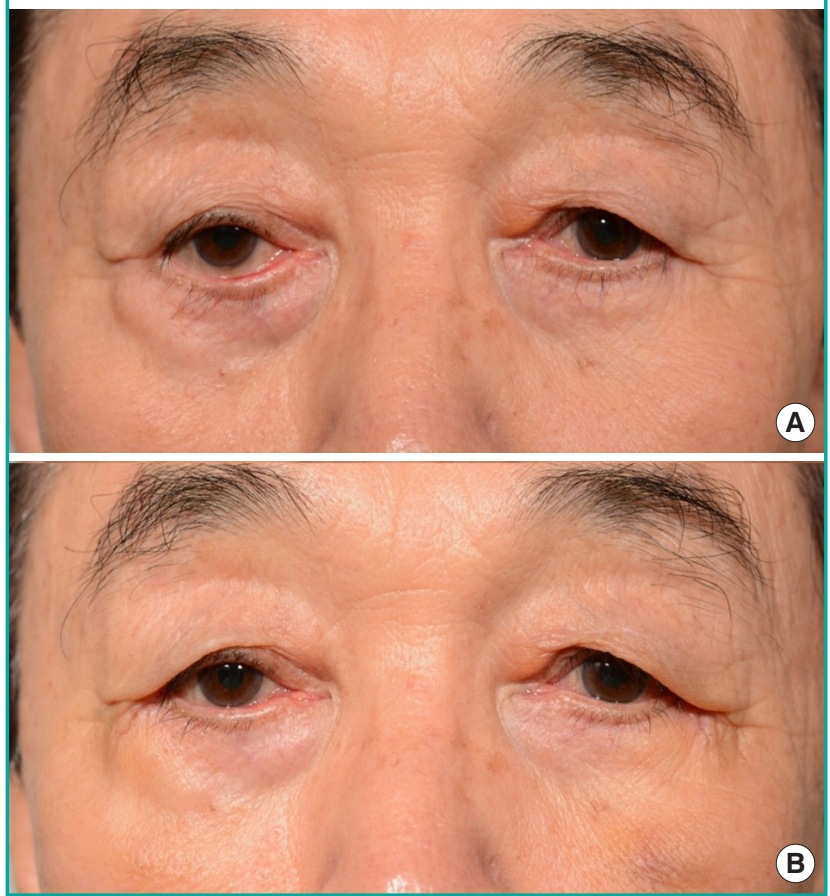

sy. In the medial canthopexy procedure, the Mitek bone anchor system was used for fixation. For lateral canthopexy, a tarsal strip procedure with a suspension suture was performed. Additionally, she had unilateral eyebrow ptosis and combined lagophthalmos; thus, we performed gold plate insertion and brow lift procedures. At 6 months postoperatively, her ectropion had resolved without recurrence (Fig. 7).

No major complications such as infections or secondary entropion occurred. There was one case of recurrent ectropion, but it resolved after an additional canthopexy procedure, and the stability of the outcome was confirmed at a 1-year postoperative follow-up.

\section{DISCUSSION}

The eye has an important impact on a person's facial aesthetic features and impression. Patients with lower lid ectropion have scleral show due to lower lid eversion. An eye with scleral show can appear sad, tired, and somewhat impassive, which can be a frustrating experience for patients. It also causes lagophthalmos of the lower lid, leading to symptoms such as eye dryness,

\section{Fig. 6. Severe cicatricial ectropion with lateral lid malposition}

(A) A 61-year-old male patient had a history of infectious disease resulting in destructive deformity on a unilateral bony orbit and the surrounding soft tissues, causing severe lateral lid malposition. He primarily underwent reconstructive surgery for the orbital bone defect using Medpor. A combined lateral canthopexy procedure using 1-0 steel-wire suspension to the lateral orbital rim, with drilling, and a full-thickness skin graft to the anterior lamella was performed. After 2 years, ectropion recurred, and tarsal strip suspension was implemented for revision. (B) After a year of follow-up since the last procedure, the outcomes remained stable and corrected.
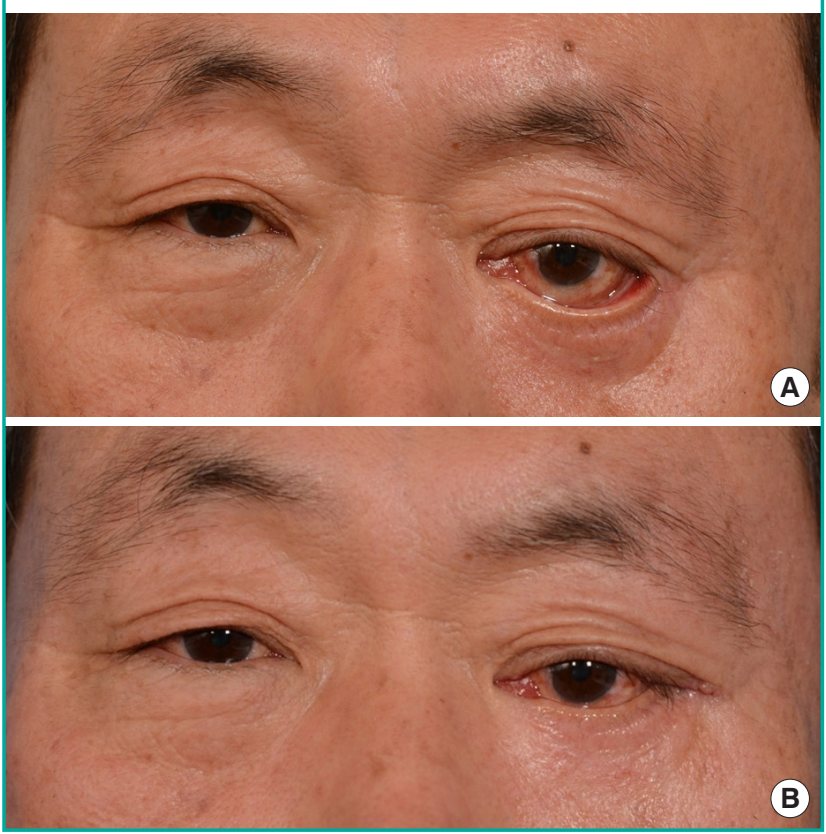

epiphora, irritation, pain, and keratopathy. Patients suffering from these symptoms should be appropriately treated.

This study proposes an algorithm that can help surgeons to manage ectropion in an easier and more logical way, in order to obtain effective results. To treat ectropion effectively, the most crucial task is to appropriately assess the cause and to classify the case as the correct type. The first step in the evaluation is to take a history from the patient and to examine the lower lid. Conventionally, ectropion is classified as paralytic, cicatricial, and involutional ectropion. However, based on our experiences, we also thought that it would be useful to consider the possibility of preventing potential or latent ectropion.

Some patients do not have evident ectropion features, but have sufficient lid laxity to develop future ectropion. Furthermore, patients who are about to undergo procedures such as lower blepharoplasty that can cause secondary ectropion should receive concomitant prophylactic treatment. Therefore, we classified cases of ectropion using a more practical and comprehensive categorization of current or present ectropion and potential or latent ectropion.

In general, the patients with latent ectropion tended to be el- 


\section{Fig. 7. Ectropion with medial and lateral lid malposition}

(A) A 69-year-old female patient had severe medial and lateral lid malposition caused by congenital unilateral complete facial palsy. For medial canthopexy, the Mitek system was used for anchorage. For lateral canthopexy, the tarsal strip procedure with a suspension suture was performed. (B) At 6 months postoperatively, ectropion had resolved without recurrence.

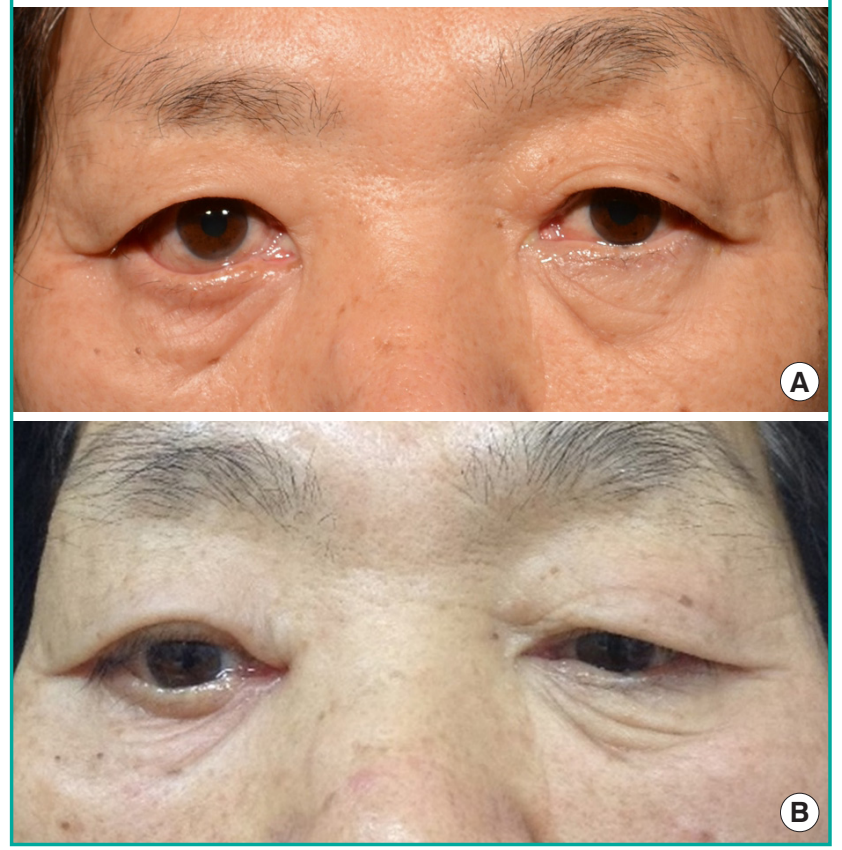

derly, and the majority of them had reduced lid tone, which can potentially cause senile ectropion. Moreover, they visited the clinic requesting lower blepharoplasty for rejuvenation, which in turn is the main cause of secondary cicatricial ectropion. Therefore, as a means of prevention, rejuvenation, and correction of mild senile ectropion, we propose performing routine combined orbicularis oculi muscle canthopexy with lower blepharoplasty. As the preseptal oculi muscle is suspended to the inferior part of the lateral orbital rim periosteum, the oculi muscle bunches up in the pretarsal area. This results in pretarsal fullness, and we were also able to achieve additional rejuvenation effects other than the basic effects of canthopexy, such as changing the canthal tilt more positively or tightening the lidcheek segment. This procedure was also reported to be effective in previous studies, one of which reported that the rate of postlower blepharoplasty lid malposition decreased from $10 \%$ to $1 \%$ after adopting the procedure [10].

If there is no evidence of current ectropion, lid laxity should be assessed in order to determine the likelihood that the patient will develop ectropion. An anterior distraction of the lower lid exceeding $6 \mathrm{~mm}$ it is considered to be a severe degree of laxity, and the structure must be hung up more rigidly and durably in order to prevent recurrence. Anderson and Gordy first de- scribed the lateral tarsal strip canthopexy, and many surgeons have modified the procedure $[11,12]$. It has several advantages over other techniques. First, both lid laxity and excessive horizontal length can be corrected simultaneously. Second, it has the cosmetic advantage of maintaining the anatomical canthal angle. Lastly, rapid rehabilitation is possible, making it suitable for elderly patients who cannot visit the clinic frequently [13].

Therefore, in patients with no evident ectropion, but only lid laxity, the lateral tarsal strip was used as the target of suspension. As a structure, it is fibrous enough to bear the force of suspension for a long period. Additionally, in patients with severe laxity, the horizontal length of the lower lid margin can be reduced through wedge resection.

In patients with evident ectropion, the displacement of the lid should be evaluated to determine the most appropriate next step. Malposition can be easily examined by comparing the location of the punctum with either the lower and upper eyelids or both lower eyelids. In cases of medial lid malposition, the caruncle is excessively exposed.

Present ectropion with lid malposition should be treated according to its pathophysiology. Generally, in cases with lid malposition, lamellar contracture is likely to be the main cause. Most such cases can be fully resolved without any additional grafts or flaps by releasing the contracture layer by layer. The structures comprising the lamella can be completely freed from the surrounding retracting tissues. However, if the anterior lamellar contracture is significant enough to result in a shortage of tissue, a full-thickness skin graft or local flap surgery should be considered to resolve the deficiency of the anterior lamella. For severe posterior lamellar contracture, a composite graft such as a palate mucosal graft should also be considered.

Despite the additional procedure to correct the lower lid deficiency, as time goes on, contracture can progress, resulting in recurrence of ectropion. Moreover, as the retracting forces are combined with gravity, more powerful tightening procedures should be used than for other types of ectropion. Thus, the structure used for suspension and the material used as the anchor should be more rigid and stronger. This can be achieved by drilling into the lateral orbital rim or using bone anchorage equipment [6]. We passed a 1-0 steel wire through the drilled hole. The advantage of using the drilling method is that it can yield an even more superior-posterior vector to the lateral tarsal margin than the original lateral tarsal strip canthopexy method. Moreover, the lower lid becomes more inverted close to the orbit, preventing irritating ectropion symptoms. Although the most ideal anchoring spot is Whitnall tubercle itself, it is difficult to approach through a minimally extended subciliary incision. Drilling the lateral orbital wall makes it possible to pass the 
steel wire through the hole from the inner to outer aspect of the lateral rim, thereby anchoring the lateral tarsal end more medially, similar to the original position of Whitnall tubercle.

In patients with medial lid malposition, medial canthopexy should be performed. We suggest that the anchoring spot should be just superior to the medial canthal tendon insertion site. This is for the same reason as in lateral canthopexy; it allows the tarsoligamentous structures to be pulled up more medially and superiorly. For medial canthopexy, we used the Mitek anchoring system to make the procedure simpler and more effective. Considering the anatomy around the medial canthal tendon, drilling a hole for canthopexy is risky. Various procedures have been developed for medial canthopexy, of which transnasal canthopexy is particularly widely known. However, it requires an extensive incision for the approach and is technically difficult [14]. We used a minimal incision for the approach; thus, we found that the Mitek Micro bone anchor system was an adequate alternative for anchoring fixation. The Mitek system is composed of a titanium alloy for anchoring onto the bone and two strands of Ethibond sutures with needles on the end. Several studies have reported that the Mitek suture anchor was advantageous for the correction of medial lid ectropion [14-17].

In addition to canthopexy procedures, other optional procedures can be performed. If a patient had been diagnosed with complete or unrecoverable incomplete palsy through electromyography, but had a responsive temporalis muscle, a facial reanimation procedure using temporalis transfer was performed along with canthopexy. Furthermore, if the patient had upper lid lagophthalmos or brow ptosis, gold plate insertion or a suprabrow lift using the frontalis suspension sling was performed together as combined surgery.

Patients were mostly satisfied with their aesthetic appearance after surgery. Prior to surgery, they were unhappy with their appearance, but after surgery, most of the patients had a score above 3 (satisfactory). Epiphora was the least improved symptom. It is presumed that even if the punctum is moved closer to the globe through the canthopexy procedure, in paralytic patients, it cannot be completely resolved because of a flaccid oculi muscle that cannot function as a lacrimal pump.

Almost all patients who visited our clinic desired the surgical procedure to be performed simply and quickly with local anesthesia. If there is no need for additional surgery, such as a facial reanimation procedure, it was possible to do so in an outpatient setting.

Although some studies have proposed algorithmic approaches for lower lid ectropion, most of them involved too extensive and aggressive procedures. Pepper et al. [7] proposed an algorithm for paralytic ectropion management that included suborbicu- laris oculi fat pad lift, which involves extensive subperiosteal dissection of the infraorbital area. In the study of Fagien [8], for some procedures in the algorithm, an additional upper transcutaneous blepharoplasty incision was necessary. In the algorithm proposed in the present study, no additional extensive dissection or incision was necessary for the canthopexy, and the procedures were effective, simple, and easy to perform.

For the adequate treatment and prevention of ectropion, it is important to decide what canthopexy procedures to perform. Medial and lateral canthopexy should be accurately adjusted. Through the suggested simple surgical algorithm in our study, both medically acceptable results and patient satisfaction were achieved without significant postoperative complications. Therefore, for management of patients with ectropion, the algorithm of this paper would be useful.

\section{NOTES}

\section{Conflict of interest}

No potential conflict of interest relevant to this article was reported.

\section{Ethical approval}

The study was approved by the Institutional Review Board of Korea University Anam Hospital (K2018-1711-003) and performed in accordance with the principles of the Declaration of Helsinki. Written informed consents were obtained.

\section{Patient consent}

The patients provided written informed consent for the publication and the use of their images.

\section{REFERENCES}

1. Bedran EG, Pereira MV, Bernardes TF. Ectropion. Semin Ophthalmol 2010;25:59-65.

2. Bergeron CM, Moe KS. The evaluation and treatment of lower eyelid paralysis. Facial Plast Surg 2008;24:231-41.

3. Vallabhanath P, Carter SR. Ectropion and entropion. Curr Opin Ophthalmol 2000;11:345-51.

4. Georgescu D. Surgical preferences for lateral canthoplasty and canthopexy. Curr Opin Ophthalmol 2014;25:449-54.

5. Azuma R, Aoki S, Aizawa T, et al. The vertical orbicularis oculi muscle turn-over procedure for the correction of paralytic ectropion of the lower eyelid. Arch Plast Surg 2018;45: 135-9.

6. McCord CD, Boswell CB, Hester TR. Lateral canthal anchoring. Plast Reconstr Surg 2003;112:222-37. 
7. Pepper JP, Kim JC, Massry GG. A surgical algorithm for lower eyelid resuspension in facial nerve paralysis. Oper Tech Otolayngol Head Neck Surg 2012;23:248-52.

8. Fagien S. Algorithm for canthoplasty: the lateral retinacular suspension: a simplified suture canthopexy. Plast Reconstr Surg 1999; 103:2042-53.

9. Trussler AP, Rohrich RJ. MOC-PSSM CME article: blepharoplasty. Plast Reconstr Surg 2008;121(1 Suppl):1-10.

10. Jacobs SW. Prophylactic lateral canthopexy in lower blepharoplasties. Arch Facial Plast Surg 2003;5:267-71.

11. Anderson RL, Gordy DD. The tarsal strip procedure. Arch Ophthalmol 1979;97:2192-6.

12. Jordan DR, Anderson RL. The lateral tarsal strip revisited: the enhanced tarsal strip. Arch Ophthalmol 1989;107:6046.
13. Olver JM. Surgical tips on the lateral tarsal strip. Eye (Lond) 1998;12(Pt 6):1007-12.

14. Antonyshyn OM, Weinberg MJ, Dagum AB. Use of a new anchoring device for tendon reinsertion in medial canthopexy. Plast Reconstr Surg 1996;98:520-3.

15. Hayashi A, Maruyama Y, Okada E, et al. Use of a suture anchor for correction of ectropion in facial paralysis. Plast Reconstr Surg 2005; 115:234-9.

16. Alfano C, Chiummariello S, Monarca C, et al. Lateral canthoplasty by the Micro-Mitek Anchor System: 10-year review of 96 patients. J Oral Maxillofac Surg 2011;69:1745-9.

17. Bartsich S, Swartz KA, Spinelli HM. Lateral canthoplasty using the Mitek anchor system. Aesthetic Plast Surg 2012; 36:3-7. 